\title{
Workshop on Computational Finance and Business Intelligence
}

\author{
Yong Shi ${ }^{1,2}$, Shouyang Wang ${ }^{3}$, and Xiaotie Deng ${ }^{4}$ \\ ${ }^{1}$ Research Center on Fictitious Economy and Data Sciences, Chinese Academy of Sciences \\ 100080 Beijing, China \\ ${ }^{2}$ College of Information Science and Technology, University of Nebraska at Omaha \\ Omaha NE 68182, USA \\ ${ }^{3}$ Academy of Mathematical and System Sciences, Chinese Academy of Sciences \\ Beijing 100080, China \\ ${ }^{4}$ Department of Computer Science, City University of Hong Kong \\ Tat Chee Avenue, Kowloon, Hong Kong \\ yshi@gucas.ac.cn, sywang@mail.amss.ac.cn, \\ csdeng@cityu.edu.hk
}

\section{Introduction}

The workshop focus on computational science aspects of asset/derivatives pricing \& financial risk management that relate to business intelligence. It will include but not limited to modeling, numeric computation, algorithmic and complexity issues in arbitrage, asset pricing, future and option pricing, risk management, credit assessment, interest rate determination, insurance, foreign exchange rate forecasting, online auction, cooperative game theory, general equilibrium, information pricing, network band witch pricing, rational expectation, repeated games, etc.

\section{Acknowledgments}

This workshop was partially supported by National Natural Science Foundation of China (Grant No.70621001, 70531040, 70501030, 10601064), National Natural Science Foundation of Beijing (Grant No.9073020), 973 Project of Chinese Ministry of Science and Technology (Grant No.2004CB720103), and BHP Billiton Cooperation of Australia. 